\title{
WHO Air Quality Guidelines: Moving Indoors
}

\author{
Kirk R. Smith
}

Received: 23 April 2008 / Accepted: 25 April 2008 /Published online: 24 May 2008

(C) Springer Science + Business Media B.V. 2008

The summary of the new WHO Global Air Quality Guidelines by Krzyzanowski and Cohen notes two important innovations in "guideline science" developed for this revision:

- Resisting the temptation succumbed to by previous committees to stop at the stage of proposing standard exposure-response functions when faced with pollutants without clear thresholds, the AQG expert group stated the new AQGs in terms of actual concentration levels that provide clear guidance to policy;

- Accommodating the challenge posed by creating "global" AQGs in the face of large differences in current pollution levels around the world, the new AQGs incorporate a tiered set of guidelines in the form of "interim targets" that enable countries to set a structured pace of control efforts over time to eventually reach the AQGs themselves.

The authors, however, did not describe a third major innovation of the new AQGs. As part of being "global," the new AQGs are meant to apply not just to outdoor environments but, for first time among WHO AQG documents, they explicitly apply to all major non-occupational microenvironments, i.e., all places where the public spends time including households, vehicles, schools, and commercial establishments. Although one might argue that there is no intrinsic reason why people should be protected differently during the part of the week when they happen

\section{K. R. Smith $(\square)$}

Global Environmental Health, School of Public Health,

University of California,

Berkeley, CA 94720-7360, USA

e-mail: krksmith@berkeley.edu to be at their jobs, the force of convention, the vast variety of occupational settings, and that other agencies deal with occupational guidelines led to the occupational exclusion.

As with workplaces, public indoor environments have sometimes also been considered intrinsically different from the outdoors and thus subject to different criteria when establishing AQGs. This led, for example, to previous WHO AQG documents specifically excluding indoor environments. The new AQG expert group recognized several reasons why this does not make sense (Smith 2006). Here, I briefly discuss two:

- Given the immense success of outdoor epidemiologic studies, which measure pollution outdoors, it is sometime forgotten that the actual exposures causing ill health occur indoors. Most of the breathing is done where the people spend time and most of people's time is spent indoors. Thus, it may be changes in outdoor levels that create changes in health, but these are mediated through indoor exposures. Although true everywhere, the relative importance of indoor and outdoor exposures varies greatly by pollutant, climate, housing, behavior, and other factors. That such factors are not and never will be known in detail for the world's population, and in addition change with development, season, and custom, means in practice that no practical distinction can be made between the levels of pollution outdoors and the exposure to those pollutants indoors.

- The alternative, different AQGs for indoors and outdoors, does not pass the "laugh test." If less stringent indoors, for example, should indoor pollution be kept inside - a chimney be a bad thing? If more stringent indoors, would opening a window be a bad thing when outdoor pollution is above the indoor limit? Would people be somehow safer by stepping out the door and 
thus suddenly be within the AQG even though their exposure would go up? People breathe indoors as they do outdoors and move between all microenvironments as they please and in different ways for different people. They want to be protected in all locations equally.

It is true that the evidence base for determining the effects of indoor sources on health are quite different in kind and quantity from the evidence base associating outdoor concentrations and sources with health. Because one or a few monitoring stations can reasonably indicate exposure variation in millions of people at once, outdoor epidemiology has the nearly unique, and highly envied, ability to determine quite small risk levels with high precision and credibility. In contrast, a measuring station indoors is likely to indicate exposure variation for just one family, i.e., some five orders of magnitude lower "station effectiveness." Thus, the fact that studies of indoor pollutant levels and health lead to less strong results can be expected - there is just not the ability to measure exposure sufficiently well across the large populations needed to do otherwise. The point remains, however, that as noted above, nearly all air pollution epidemiologic studies are actually studies of indoor exposures no matter where the measurements are made.

Although progress was made in dealing with three difficult issues in AQG setting, the new guidelines do not address others. Among the most difficult is the problem posed by establishing guidelines across pollutants in a consistent manner. Does it make sense to set each AQG based solely on evidence about its own effects disregarding the incremental improvement in health involved? Arguably, this tends to set AQGs on the basis of how many studies happen to have been done for a pollutant rather than to guide rational allocation of protection efforts? For example, in the new guidelines, the incremental impact on health is substantially different across ozone and PM2.5 at the 24-h AQG for each, whether measured in absolute or relative terms. A number of issues would have to be considered to choose the best approach, but it seems clear that the current pattern on independent assessments can lead to misrepresentation of the overall air quality situation and thus potentially to misallocation of resources when used, for example, in a pollution standards index, which is a common practice worldwide.

\section{References}

Smith KR (2006) Four discussion pieces on setting air quality guidelines. Proceedings of the Health Effects Institute Annual Meeting, HEI, Boston, MA. http://ehs.sph.berkeley.edu/krsmith/ page.asp?id $=5 \# 7$ 